\title{
Patrones de cobertura periodística: frames genéricos y frames de nivel en las elecciones presidenciales peruanas del 2016
}

Journalistic coverage patterns: generic frames and level frames at the 2016 Peruvian presidential elections

SANDRO MACASSI LAVANDER

Psicólogo social y magister en Comunicación. Profesor de la Pontificia Universidad Católica del Perú. Se ha especializado en "conflictos y comunicación preventiva". Ha sido consultor en temas de comunicación para el desarrollo para el Programa de las Naciones Unidas para el Desarrollo (PNUD), la Organización Panamericana de la Salud (OPS), el Chemonics Inc., el Banco Interamericano de Desarrollo (BID), el World Bank, el Netherlands Development Organisation (SNV) y el Ministerio de Economía y Finanzas (MEF). Además, ha desarrollado investigaciones sobre medios, periodistas y comunicación en conflictos socioambientales. 



\title{
Patrones de cobertura periodística: frames genéricos y frames de nivel en las elecciones presidenciales peruanas del 2016 Journalistic coverage patterns: generic frames and level frames at the 2016 Peruvian presidential elections
}

\author{
Sandro Macassi Lavander \\ PONTIFICIA UNIVERSIDAD CATÓLICA DEL PERÚ \\ semacassi@pucp.pe
}

\section{PALABRAS CLAVE / KEYWORDS}

Frames genéricos / frames de nivel / frame de conflicto / elecciones presidenciales, análisis de contenido

Generic frames / level frames, conflict frame / presidential elections, content analysis

\section{SUMILLA}

En este texto se hace una revisión de la literatura sobre análisis de frames, poniendo atención en la relación entre frames y política en procesos electorales.

Se plantea también un análisis de dos tipos de frames: los de nivel y los genéricos, haciendo una comparación de aquellos que fueron presentados en las noticias de dos elecciones peruanas, la del 2014 y la del 2016, haciendo hincapié en las elecciones presidenciales del 2016. De igual modo, se analiza si hay diferencias entre los tipos de medios. Para ello se registraron 5086 noticias electorales de 20 programas y espacios noticiosos de radio, prensa y televisión.

De lo encontrado se concluye que si bien existe un patrón de cobertura de uso de frames que persiste de una elección a otra, este difiere durante las fases de primera y segunda vuelta de la campaña electoral.

El uso frecuente del "frame de moralidad" constituye un sello particular de la cobertura periodística peruana, aunque también sea habitual que utilicen "frames de conflicto”, estos últimos predominantes a nivel mundial en los contextos electorales. Por tanto, la cobertura de los medios peruanos sigue patrones de producción globalizados aunados con esquemas propios de la cultura periodística nacional, generando una hibridación de patrones de producción noticiosa.

\section{ABSTRACT}

This text reviews the literature on frame analysis, paying attention to the relationship between frames and politics in electoral processes. The studies on 
level frames and on generic frames are discussed.

The types of frames are analyzed: level frames and generic frames. The use of both frames is compared in the Peruvian elections of 2014 and 2016, emphasizing the presidential elections of 2016. Likewise, it is analyzed if the use of these frames show differences between the types of media.

The study was based on the analysis of 5086 electoral news from 20 programs and radio, press and television news spaces.

The findings show that there is a pattern of coverage of the use of frames that persists from one election to another. But, there are differences in the use of frames from the first phase to the second round of the electoral campaign.

Like the studies on coverage in other countries, in the Peruvian case the use of "conflict frames" is common, However, it differs from the international pattern because in the Peruvian case there is a more frequent use of the "frame of morality". This is a particular feature of Peruvian newspaper coverage.

Therefore, the coverage of the Peruvian media follows globalized production patterns together with schemes typical of the national journalistic culture, generating a hybridation of news production patterns.

\section{Presentación}

La preocupación por el rol que cumplen los medios de comunicación en los procesos de significación política es cada vez más vigente en Latinoamérica, toda vez que nuestros países atraviesan grandes dificultades de representación política. A esto se le suma el hecho de que son los medios de comunicación quienes delimitan las reglas de la política, producto de la crisis de los sistemas de partidos (Strömbäck, 2008). Por tanto, usualmente los medios se desempeñan como actores políticos con gran independencia (Manin, 2006), además de funcionar como reguladores del sistema político (Programa de las Naciones Unidas para el Desarrollo [PNUD], 2004), recurriendo para ello a estrategias de construcción de las noticias como los frames, los atributos o la agenda setting, que catalizan la política.

La literatura sobre la relación entre medios de comunicación, política y la incidencia en los ciudadanos es vasta. En la última década se ha prestado particular atención a la forma en cómo los medios presentan las noticias, analizando la relación que establecen entre su estructura, contenidos e interpretación. Las teorías que abordaron eficazmente el estudio de dicha relación han recibido el nombre framing theory o "teorías del marco".

Como bien lo anotan Semetko y Valkenburg (2000): "Framing analysis shares with agenda setting research a focus on 
the relationship between public policy issues in the news and the public perceptions of these issues". Sin embargo, las teorías del framing van un paso más allá que la agenda setting, pues no se limita a señalar solamente cómo se seleccionan los temas que se discuten y piensan (agenda), sino que se enfocan en la manera en que estos son resaltados por los periodistas.

Sin embargo, a diferencia de los estudios sobre agenda, los estudios sobre frames, como lo señala Scheufele (1999), constituyen un archipiélago heterogéneo de teorías y mediciones que no tienen un cuerpo teórico central. Por lo tanto, no existe una definición única de los frames, y más bien se trata de un paradigma fragmentado (Entman, 1993). Según Matther (2012), es necesario desarrollar una teoría que integre los diferentes estudios con una perspectiva interdisciplinaria.

\subsection{Los frames como objeto de estudio}

Goffman (1986) fue uno de los primeros en definir los frames como "principios de organización que gobiernan los eventos y nuestro involucramiento subjetivo en ellos”, desde el punto de vista de la observación y de la interiorización de los contenidos. Posteriormente, diversos autores desarrollaron conceptualizaciones de los frames aplicados principalmente a la producción noticiosa.

Muchas definiciones coinciden en que los frames hacen referencia a reglas o patro- nes de organización, o estructuración, de la información que se usa para comprender la realidad, o comunicarla, de tal manera que genere sentido a un conjunto de acontecimientos.

Gamson y Modigliani (1989, p.106), a nuestro juicio, definen acertadamente los media-frames, diferenciándolos de la definición de Goffman, argumentando que los frames son "a central organizing idea or story line that provide meaning to an unfolding strip of events".

Por su parte, Scheufele (1999, p.106) formuló una clasificación para comprender el archipiélago de producción investigativa sobre los frames. Este autor identifica tres corrientes: una primera que sitúa el análisis en la construcción de los frames (frame building); la segunda que sitúa el análisis en la estructura de sentido que tienen las producciones comunicativas (media frames); y la tercera que sitúa a los frames en la interpretación de los públicos o frames individuales (audience frames).

Respecto al frame building, Gitlin (1980, p.7) sostiene que es un proceso clave en las rutinas periodísticas, donde se utilizan "patrones frecuentes de cognición, interpretación y presentación de selección, énfasis y exclusión que rutinariamente organizan el discurso”. Además, para los periodistas, al momento de elaborar el discurso noticioso, los frames les permiten organizar cognitivamente los hechos y sus contextos. 
En el análisis de los media frames, Entman (1993) observa que estos contienen una "lectura preferente" que es promovida por la estructura gramatical de las noticias, que se construye a través de la selección y combinación de recursos comunicativos, buscando un efecto predominante en la audiencia. Así pues, cuando analizamos los media frames no solo observamos la estructura el discurso, sino que analizamos una definición del problema, identificamos las causas, los juicios de valor y el curso de acción propuesto en el texto (Entman, 1993).

La corriente de autores que han puesto énfasis en el análisis de los frames de interpretación o audience frames (Scheufele e Iyengar, 2017; Busby, Flynn y Druckman, 2018) sostienen que a los públicos los individual frames les facilitan la organización y síntesis de la información, en base al dominant meaning que se comparte culturalmente, aunque no se descartan interpretaciones alternativas (Entman, 1993, p.56).

Sin embargo, estos tipos de frames no deben verse como procesos inconexos. Neuman, Just y Crigler (1992), por ejemplo, ponen énfasis en el proceso comunicativo que los frames facilitan, donde tanto periodistas como audiencias cuentan con códigos para convenir, interpretar y evaluar la información. Por su parte, de Vreese (2005) sostiene que la interacción entre la producción de frames (frame building), interpretación individual de la audiencia (frame setting) son un proceso dinámico. Luego de este proceso se construyen colectivamente los discursos políticos, institucionales y los atajos cognitivos interpersonales.

Semetko y Valkenburg (2000) afirman que los frames son un proceso cultural ya que sus códigos de elaboración e interpretación son construcciones en torno a acontecimientos políticos, producto de procesos de flujo entre los procesos productivos y los receptivos. Para Gamson y Modigliani (1989, p.3) los media frames se constituyen en "paquetes interpretativos que le dan sentido a un asunto", vinculando emociones e informaciones a los hechos, que a fuerza de repetirlos en el tiempo se incorporan al sentido común que da forma a la cultura popular sobre la política. En cambio, para Capella y Jamieson (1997, p.47) lo que hacen los frames es "activar el conocimiento, estimular el stock cultural de costumbres y valores y crear contextos”, influyendo en cómo los ciudadanos procesan los hechos noticiosos y entienden los fenómenos políticos.

\subsection{Frames periodísticos y su rol político}

Acertadamente D`Angelo (2018) hace una diferencia entre los media frame y los news frames. Originalmente los estudios se centraron en las noticias (news), pero con el tiempo surgieron investigaciones sobre los frames en otros géneros 
de producción mediática (media), diferentes al género informativo. En este análisis nos ocuparemos del estudio de los news frames.

En la producción periodística se emplean los frames para simplificar la elaboración de noticias, estructurando los acontecimientos emergentes de una manera rápida y sencilla, identificando y clasificando las informaciones para convertirlos en hechos noticiosos.

Sin embargo, los frames implican estrategias comunicativas de relacionamiento con las audiencias. Por ejemplo, cuando en una noticia se acentúa un frame de interés humano sobre un determinado acontecimiento, el periodista apunta a que la audiencia se relacione desde la solidaridad y la emocionalidad. En cambio, si el mismo acontecimiento es construido desde un frame de consecuencias económicas, se espera que la audiencia reaccione más racionalmente, preocupándose de los impactos del hecho en la economía.

Entman (1991) plantea que los news frames cumplen cinco funciones básicas para la comprensión de los hechos periodísticos por parte de las audiencias. Estos son:

Definir los problemas, presentando los obstáculos surgidos a curso de acción y definir sus dimensiones.

Diagnosticar causas, que identifican las fuerzas que crean el problema.
Identificar a los actores relacionados al problema y adscribirlos a determinados atributos.

Evaluar las intenciones de los agentes o sus objetivos, prescribiendo sus conductas o calificándolas.

Ofrecer y justificar el abordaje público de los problemas y predecir sus impactos.

Al respecto, Kiousis (2005) señala que "los medios no solo nos cuentan sobre qué pensar, ellos también pueden contarnos cómo y qué pensar acerca de los temas y, más aún, qué hacer al respecto”. Esta premisa implica una dimensión política que ha sido una preocupación transversal del análisis de los frames.

Las investigaciones demuestran que determinados usos de frames buscan mantener el statu quo simplificando los problemas y despojándolos de los aspectos cuestionadores (Scheufele, 1999). Además, tienden a priorizar los sentidos del mainstream (Tuchman, 1973), excluyendo los enfoques alternativos (Gitlin, 1980).

Los frames también fueron usados para dar legitimidad a las acciones gubernamentales durante las guerras, reforzando un clima de amenaza externa, fomentando el patriotismo y justificando las acciones bélicas (Gamson y Modigliani, 1989).

Por su parte, Newman et al. (1992) encuentran que el uso de determinado 
frame asociado repetidamente a cierto acontecimiento o actor generan una estereotipación o estigma. Por ejemplo, cuando los sindicatos son presentados constantemente con el frame de confrontación o conflicto se promueve su estereotipación como organizaciones problemáticas.

También existe la preocupación por un tipo de cobertura mediática que presenta las elecciones como una carrera de caballos en detrimento de una cobertura de la substancia de los contenidos políticos, fenómeno que se viene intensificando elección tras elección (Patterson, 1994). Enfocar la política como una competencia o una guerra se ha convertido en permanente debido a la incesante narrativa de quién queda adentro y quién queda afuera, degradando la credibilidad de la política (Blumler y Coleman, 2010, p.143), presentándose este enfoque con mayor intensidad en los medios online que en los offline (Schmuck, Heiss, Matthes, Engesser y Esser, 2016).

Otros autores señalan que esta forma de cubrir las elecciones (como competencia/ guerra) es el resultado del uso de frames de juego-estratégico (Capella y Jameison, 1997; Iyengar, 1994; de Vreese 2005) debido a que estos impactan negativamente en la calidad de la información de los ciudadanos y, por tanto, en los procesos deliberativos públicos. Así mismo, el uso de frames de juego-estratégico influye en el sistema de partidos y sus instituciones (Dimitrova y Kostadinova, 2014).

\section{Frames de nivel y frames genéricos en procesos electorales}

En cuanto al uso de frames en procesos electorales, tenemos una abundancia de estudios al respecto. Algunas investigaciones centran su preocupación en el efecto atomizador que la cobertura tiene sobre las campañas electorales, frente a la necesidad de que la cobertura se centre en lo programático.

Gamson, Ccroteau, Honey y Sansom (1992, p.385) hacen una distinción importante, señalando que existen dos niveles de enmarcamiento en las noticias. Ellos identifican el frame-event como aquel que está circunscrito a un acontecimiento, como puede ser un accidente en una planta nuclear. A este frame de Vreese (2005) lo llama: issue-specific news frames o frame episódico.

En contraste con el frame episódico, Gamson et al. (1992) señalan que existe un frame-issue, que enmarca una interpretación en la cual el accidente es parte de una cadena de eventos y acciones como puede ser la seguridad de las plantas nucleares.

Sin embargo, la tipología propuesta por estos autores no marca una diferencia entre un frame que aborda políticas públicas, como puede ser la política energética gubernamental, de asuntos como la seguridad ciudadana En un proceso 
electoral discurren ambos tipos de nivel de temas, asuntos como la corrupción o la cuota paritaria de mujeres, pero su abordaje puede ser como promesas generales de buena voluntad o propuestas concretas de políticas públicas. En ese sentido, la propuesta de Gamson et al. (1992) requiere de una mayor precisión respecto a las políticas públicas o directrices de gobierno, diferenciándolas de las situaciones políticas.

Iyengar (1994) sostiene que el uso predominantemente de frames episódicos ha transformado los procesos electorales, pues en foco de la discusión está en la cotidianeidad de las actividades proselitistas. La sobrepresencia de frames episódicos tiene como consecuencia que desenfoca la atención sobre los contenidos políticos (thematic frame) y la construcción de sentidos colectivos. Por tanto, a los ojos de las audiencias el proceso electoral aparece como un conjunto de actividades sensacionalistas, sin las claves interpretativas para entender integralmente las propuestas de los candidatos.

De otro lado, de Vreese (2005) clasifica como frames genéricos a una línea de investigación de los frames en procesos electorales, donde se incluye tanto a los frames de juego-estratégico (Capella y Jamieson, 1997; Muñiz, 2015; Alberg et al., 2011), descritos líneas arriba, como a un grupo de frames que reflejan la organización de producción periodística.
Neuman et al. (1992), basándose en entrevistas a la audiencia, identificó cinco tipos o estructuras de frames que los públicos usan para convenir, interpretar y evaluar la información política. Estos son: frame económico, frames de conflicto, frame de indefensión, frame de impacto humano y frame de moralidad. Basados en estos frames, Semetko y Valkenburg (2000) desarrollaron una tipología para el análisis de los discursos en las noticias: frames de conflictos, de consecuencias económicas, de interés humano, de moralidad y de atribución de responsabilidad.

Cabe recalcar que la tipología de Semetko y Valkenburg (2000) refleja un acento en las estructuras de los discursos, estando más preocupados en encontrar patrones de producción periodística. La tipología propuesta no excluye la posibilidad de analizar los sentidos construidos, de hecho Chong y Druckman (2007, p.107) subrayan que al interior de cada tipo se puede analizar los significados que los grupos de poder y las líneas editoriales se esfuerzan por unificar.

Metodológicamente, tanto los frames de nivel como los frames genéricos ponen el acento en su estructura y en su influencia cognitiva en el mediano plazo. Para Gamson y Modigliani (1989), los frames son expresiones de sentidos, dándole menos importancia a su estructura, poniendo el acento en los ángulos 
o saliencia ${ }^{1}$, o, en otras palabras, en el impacto a corto plazo y en la interpretación que la audiencia hace sobre determinada problemática.

Ambos, los frames de nivel y los frames genéricos, a juicio de Kozman (2016), constituyen dos acercamientos que revelan dos funciones diferentes de las noticias. El segundo centrado en la saliencia de un asunto específico y el otro enfocado en los valores periodísticos.

Semetko y Valkenburg (200o) encuentran que los frames genéricos se diferencian según los asuntos que abordan. Por ejemplo, en las disputas sobre el euro se usan más frames de responsabilidad y de consecuencias económicas, mientras que en la cobertura sobre un crimen se usan más frames de conflictos y de interés humano.

Algo similar encuentran de Vreese, Peter y Semetko (2001), pues los frames de conflicto y de consecuencias económicas difieren cuando se trata de asuntos políticos o cuando es sobre temas económicos (como por ejemplo sobre la introducción del euro a la economía europea). De esta manera, puede entenderse que no solamente existen diferencias en el uso de frames entre los medios, sino que los que se utilizan también se diferencian según el tipo de asuntos cubierto.
En Latinoamérica son pocos los estudios que han analizado cómo los medios hacen uso de los diferentes tipos de frames para lograr sus objetivos mediáticos. Uno de los primeros estudios longitudinales (10 meses) realizados sobre frames es el de Macassi y Graham (2003), quienes encuentran que en las noticias televisivas peruanas prima el uso de frames de conflicto $(90$ segundos, 33\%; América Noticias, 34.1\%; y 24 Horas, 43.4\%), mientras que en la radio y la prensa se utilizan estos con menor frecuencia (El comercio, 16.8\%; y RPP, $20.5 \%)$.

De igual modo, el frame de interés humano también estuvo más presente en la televisión ( 90 segundos, 11.1\%; América Noticias, 23.4\%; y 24 Horas, $14.7 \%$ ) que en la radio o la prensa $(4.6 \%$ y $2 \%$, respectivamente). De lo expuesto se puede deducir que los frames de conflicto y de interés humano se prestan más a la plataforma televisiva, probablemente debido a que la escenificación de la confrontación y el drama atraen más la atención de los ciudadanos.

En Argentina, Aruguete (2010) analizó el uso de frames en la cobertura de la privatización por tres meses de diarios impresos. Ella encuentra que los frames más frecuentes en dicho contexto fueron el de conflicto, el de consecuencias económicas y el de resolución de conflictos. Pero la intensidad de estos frames se di-

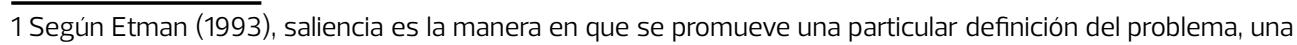
interpretación de las causas, una evaluación moral y/o recomendación sobre su abordaje, respecto al asunto descrito. 
ferenciaba en función de los asuntos que comprendían: la modalidad de la privatización, la cuestión tarifaria, la calidad del servicio, etc.

La investigación sobre frames genéricos generalmente abordó coyunturas específicas o semanas previas a la votación en procesos electorales. En el estudio de Muñiz (2015) se compararon medios impresos, sin contrastarlos con otros tipos de medios (televisión, radio, web).

En el presente estudio abordaremos la cobertura periodística que los medios de Lima realizaron del proceso electoral de las elecciones locales del 2014 y de primera y segunda vuelta en las elecciones presidenciales del 2016, identificando si hicieron un uso diferenciado de los frames genéricos y de los frames de nivel.

\section{Metodología}

Este estudio se ocupa de cómo los frames de nivel y los frames genéricos son usados de manera diferente en todo el ciclo del proceso electoral. Otros estudios analizan las etapas electorales en tres: la etapa de presentación de candidatos, de debate y de "carrera de caballos" (Muñiz, Saldierna y Marañón, 2018). Para este estudio analizaremos la elección presidencial del 2016, marcada esta por la configuración electoral de dos etapas: la primera y la segunda vuelta, haciendo una comparación del uso de los tipos de frames por los medios de comunicación en cada una estas.
Usualmente los estudios sobre los frames genéricos abarcaron periodos cortos de tiempo, sobre todo circunscritos a las semanas previas a la votación. A diferencia de los procesos electorales europeos, las elecciones peruanas son largas. La elección presidencial del 2016, por ejemplo, ocupó cerca de tres meses, abarcando el presente estudio buena parte de todo ese periodo.

Así mismo, para algunos análisis contrastaremos la cobertura de las elecciones del 2016 con las elecciones municipales del 2014, para poder establecer la existencia de una tendencia en el uso de estos frames a lo largo del tiempo.

Para el análisis se elaboraron dos herramientas: un libro de códigos y un manual de codificación. Se convocaron a estudiantes de comunicaciones para realizar el análisis de las noticias, quienes fueron entrenados en los aspectos metodológicos y teóricos del diseño. Los codificadores realizaron el análisis en formularios implementados en la plataforma online Surveymonkey. Una vez culminada la codificación, la base de datos fue exportada al SPSS v23 para el análisis de su consistencia y su posterior procesamiento.

\section{Definición de variables}

\section{a.- Frames de nivel}

Para el análisis del frame de nivel nos basamos en la definición desarrollada 
por Gamson et al. (1992), sin embargo, hicimos una modificación en el frame de asuntos (issue frame), desdoblando el indicador en dos: frame de asuntos y frame de políticas. Para su medición se consideraron que estos subtipos de frame eran mutuamente excluyentes. Además, se utilizaron las siguientes definiciones operacionales:

\section{Frame episódico}

Se enmarcan como actos o eventos aislados. Se presenta el hecho sin vinculación con otros hechos, es un acontecimiento aislado, aunque puede tener mucho detalle. Por ejemplo, se centra en las actividades proselitistas o en el hecho de que un candidato haya declarado públicamente sin presentar los contenidos de su declaración.

\section{Frame de asuntos}

Enmarcan un hecho noticioso como pertenecientes a un conjunto de hechos conectados entre sí, bajo una categoría o asuntos (issues). El hecho es relacionado a otros hechos similares, estableciendo una similitud o continuidad que configura temas de atención o atribuyendo una argumentación "de causas" o de "consecuencias".

\section{Frame de políticas}

Se enmarcan como problemáticas temas de gobernabilidad bajo un relato más amplio o macrotema. En elecciones, se trata de la referencia que se hace a políticas públicas, líneas directrices de gobierno o un conjunto de asuntos que son englobados como parte de las tareas futuras de conducción gubernamental. Puede existir una mayor elaboración, incluyendo responsabilidades o juicios morales a los actores.

\section{b.- Frames genéricos}

Para la tipología de los frames genéricos usamos la clasificación desarrollada por Semetko y Valkenburg (2000), pero basados en las definiciones de Neuman et al. (1992). Además, adicionamos dos nuevos frames, uno que titulamos "frame de diálogo" y el segundo llamado "frame de peligro", pues, como lo sostiene Aruguete (2010), la formulación de los frames genéricos deben de adaptarse a las características culturales y a los sistemas políticos que se observan.

\section{Frames de confrontación (conflictos)}

Constituye uno de los frames más frecuentemente utilizados en la comunicación política, siendo el conflicto parte sustantiva de la democracia (Bartholome, Lecheler y de Vreese, 2017). El frame de confrontación prioriza la contraposición y la disputa entre dos o más ideas, personas o instituciones. En la presentación de los hechos se resaltan los elementos que dividen y separan a los actores sociales 
en lugar de aquellos que son puntos en común. Se presenta a los actores como en una disputa entre quién gana y quién pierde (Hakansson, 2000). No necesariamente está referido a hechos de conflicto, como peleas, violencia o guerras.

\section{Frames de interés humano}

Este es uno de los frames más usados debido a que prioriza la dimensión emocional, los impactos en las personas, en sus condiciones de vida, ya sean estos tanto personales como familiares. Se da prioridad, por ejemplo, al testimonio y a la opinión de los afectados de los acontecimientos, recurriendo usualmente a la victimización de los actores. Para ello es muy común que se enfatice el llanto y el dolor.

En ciertas ocasiones este tratamiento suele individualizar los hechos, presentándolos como asuntos personales, desvinculados de los temas públicos. En un contexto de competencia, muchos editores y periodistas echan mano de este frame para capturar la mayor cantidad de audiencia y retenerla. Lo mismo sucede en la cobertura de las elecciones, cuando presentan a los candidatos, sus familias, sus hábitos o sus aficiones.

\section{Frames de moralidad}

Se construye con la calificación o juzgamiento moral o ético de los hechos o de los actores. El ángulo o saliencia suele presentar opiniones, comentarios o adjetivaciones que suponen dicho juzgamiento. Ciertamente, existen algunos temas programáticos como la libertad sexual, la despenalización del consumo de marihuana, etc., que generan más juicios morales de parte de los medios. A veces está presente a modo de conclusiones o moralejas que hacen los lectores de noticias sobre algún contenido presentado en el reportaje. También se recurre a la opinión de los grupos expertos para anunciar juicios morales sobre los candidatos o su comportamiento con el objetivo de deslegitimarlos.

\section{Frames de consecuencias económicas}

Originalmente este frame fue presentando por Neuman et al. (1992) como el énfasis que los medios hacen en las consecuencias económicas de algún problema o acontecimiento público. Comúnmente los medios resaltan las pérdidas económicas, los impactos en el bolsillo del contribuyente, las afectaciones que ciertas candidaturas o propuestas tendrían en la economía del país, o en el alejamiento de las inversiones, etcétera.

\section{Frames de responsabilidad}

El ángulo desde el que se estructura este frame enfatiza la atribución de responsabilidad en personas en lugar de realizar un análisis sistémico. Los textos inciden en la identificación de los culpables, los responsables o los involucrados en los 
acontecimientos (Iyengar, 1994). Usualmente se busca que las indagaciones los identifiquen y que precisen su grado de involucramiento. Algunas noticias incluyen un tono de sanción simbólica en la construcción del frame.

\section{Frame de diálogo}

Originalmente Neuman no formuló este frame. Sin embargo, como lo sostienen algunos autores, la existencia de frames de confrontación (conflicto) conlleva a la presencia de frames de paz (Nicolás, 2011, p.8). No obstante, el término "paz" alude a una situación posterior a la guerra y no refleja las dinámicas que se desarrollan en un proceso de deliberación electoral. Aruguete (2010) creó el frame "resolución de conflictos" para asuntos donde resolver el conflicto requiere de dos perspectivas diferentes o la actuación de terceros. En este estudio usaremos el "frame de diálogo" debido a que se trata de una formulación más amplia y que se adapta tanto a contextos electorales como a asuntos controversiales, como los que Aruguete estudia. Lo definimos como la saliencia o ángulo con que una noticia estructura el sentido de la búsqueda de soluciones, acuerdos, puntos de común, intercambios o consensos.

\section{Frame de peligro}

Debido a las características de las elec- me para dar cuenta de la polarización existente. Usualmente, ante la presencia de un candidato populista de derecha (como Keiko Fujimori en el 2011 y el 2016) y la presencia de candidaturas de izquierda (como Ollanta Humala en el 2011 y Verónica Mendoza en el 2016), los medios generan discursos catastróficos. Estas narrativas podemos enmarcarlas en un frame de peligro, donde se presentan situaciones futuras de alerta o riesgo, incentivando el miedo y la preocupación.

Finalmente, es importante mencionar que todos los frames utilizados fueron medidos dicotómicamente como presencia o ausencia de una o más características, en lugar de una escala o una variable multidimensional, debido a que priorizamos la comparación y el análisis de 10 variables durante tres meses, en lugar de un análisis más profundo de menos variables y un menor tiempo cubierto.

\section{Preguntas de investigación}

El presente estudio busca indagar sobre los patrones de continuidad en el uso de los frames entre una elección y otra (2014 y 2016). Además, averigua cómo el periodismo usa los frames en las diferentes fases electorales, primera y segunda vuelta, del proceso electoral presidencial peruano del 2016.

Las preguntas de investigación son las siguientes: 
1.- ¿Existirá una tendencia en la producción noticiosa de frames genéricos y de nivel que se mantienen constantes entre diferentes procesos electorales?

2.- ¿Las fases de primera y segunda vuelta electoral marcan diferencias significativas en el uso del frame de nivel y de los frames genéricos en las elecciones presidenciales del 2016?

3.- ¿Existen diferencias entre los medios en la frecuencia del uso de frames genéricos y de los frames de nivel?

4.- ¿Los frames genéricos y los frames de nivel estarían pauteando una cobertura centrada en lo estratégico en detrimento de la deliberación pública?

\section{Muestra de medios y periodo de observación}

Se seleccionaron 20 programas noticiosos e informativos de 12 empresas de radio, prensa y televisión de alcance nacional ${ }^{2}$. La mayor cobertura que los medios impresos dieron al proceso electoral generó que en la muestra exista más noticias de prensa que del resto de medios. Esto sucedió a pesar de que los diarios impresos fueron menos (4) que los programas televisivos (13).

El procedimiento se inició con la identificación y registro de las noticias electorales publicadas o transmitidas en los tres meses que duró la elección. Así, se contabilizaron y analizaron 5086 noticias sobre temas electorales.

La muestra consistió en 32 días de cobertura para la primera vuelta electoral y 33 días para la segunda vuelta. En la figura 1 se puede observar el número de noticias analizadas para cada uno de estos dos periodos.

Además, se usó una data de contraste de las elecciones generales del 2016 por

Figura 1. Número de noticias analizadas por periodo (elecciones generales 2016)

\begin{tabular}{|c|c|c|c|c|c|}
\hline \multirow{2}{*}{} & \multicolumn{2}{|c|}{$\begin{array}{c}\text { Primera vuelta } \\
\text { Del 11 marzo al } \\
12 \text { de abril del 2016 }\end{array}$} & \multicolumn{2}{|c|}{$\begin{array}{c}\text { Segunda vuelta } \\
\text { Del 2 de mayo } \\
\text { al 5 de junio del 2016 }\end{array}$} & \multirow{2}{*}{ Total } \\
\cline { 2 - 5 } & Frecuencia & $\%$ & Frecuencia & $\%$ & \\
\hline Noticias analizadas & 3050 & 59.9 & 2036 & 40.1 & 5086 \\
\hline
\end{tabular}

2 La muestra consistió en las siguientes empresas y programas. TELEVISIÓN. América Televisión: América Noticias Primera Edición, América Noticias Edición Central y Cuarto Poder. Frecuencia Latina: 90 Matinal, 90 Edición Central, Punto Final y Sin Medias Tintas. ATV: ATV Noticias AM, ATV Noticias FM y Día D. Panamericana Televisión: Buenos Días Perú, 24 Horas Edición Central y Panorama. TV Perú: TV Perú Noticias, TV Perú Noticias y TV Perú 7.3. RADIO. RPP: La Rotativa del Aire, Ampliación de Noticias y Enfoque de Los Sábados. Radio Capital: Phillip Butters y Aldo Mariátegui. Radio Exitosa: Nicolás Lúcar y Juan Carlos Tafur. Radio Nacional: Nacional en la Noticia y Diálogo Abierto. DIARIOS. Correo, El Comercio, Exitosa, La República, Perú 21 y El Trome. 
la elección del municipio provincial de Lima ocurrida el año 2014. Las noticias analizadas para la elección municipal fueron 209 de radio y televisión, pertenecientes a ocho espacios noticiosos de seis empresas mediáticas. Las variables analizadas en el estudio de la elección local fueron las mismas a las empleadas en las elecciones presidenciales.

\section{Discusión de resultados}

Contrastando los resultados de las elecciones presidenciales del 2016 con las locales del 2014, observamos que en ambas se hace muy poco uso de frames de políticas, por tanto, el patrón de producción de frames estaría más centrado en levantar los hechos desde un ángulo episódico y de asuntos (ver la figura 2). Esta tendencia refuerza la idea de que la cobertura periodística no prioriza los asuntos de fondo de la gobernabilidad, abordando las problemáticas pero desvinculándolas de sus implicancias de gestión pública.

Una diferencia sustantiva entre los procesos analizados es que en las elecciones municipales hay un mayor uso de frames episódicos, mientras que en las elecciones presidenciales hay una mayor presencia de frames de asuntos. Esto puede deberse presumiblemente a que en las elecciones presidenciales se presta mayor atención a los planes de gobierno y propuestas.

Si analizamos con más detenimiento los resultados de las elecciones presidenciales tenemos que se usan más frames sustantivos que frames episódicos (ver la figura 2). Es decir, que hay una mayor dedicación de la atención pública a los temas en los procesos electorales. No obstante, hay que señalar que el volumen de noticias episódicas es significantemente alto, expresando la atención que se pone en actividades de campaña que no ayudan, necesariamente, a la deliberación ciudadana que debiera estar presente en toda elección.

En las elecciones presidenciales del 2016, si analizamos las diferencias entre la primera vuelta y la segunda, vemos que en la segunda vuelta hay un incremento de los frames de asuntos y una disminución de los frames episódicos (ver la figura 3). Creemos que esto se debe a que en segun-

Figura 2. Frame de nivel según proceso electoral

\begin{tabular}{|l|c|c|c|c|}
\hline & \multicolumn{2}{|c|}{ Presidenciales 2016 } & \multicolumn{2}{c|}{ Municipal 2014 } \\
\hline & Frec. & $\%$ & Frec. & $\%$ \\
\hline Frames episódicos & 2158 & 42.4 & 104 & 49.8 \\
\hline Frames de asuntos & 2665 & 52.4 & 85 & 40.7 \\
\hline Frames de políticas & 263 & 5.2 & 20 & 9.6 \\
\hline Total & 5086 & 100.0 & 209 & 100.0 \\
\hline
\end{tabular}


da vuelta solo hay dos candidatos, por lo que la demanda de cobertura de actividades proselitistas disminuye.

Sin embargo, se esperaría que conforme se acerca el día de la votación final, debiera de haber una mayor preocupación por las líneas directrices de gobierno. En cambio, observamos que la cobertura de los frames de políticas disminuye.

En cuanto a la presencia de los frames genéricos en las elecciones presidenciales, tenemos que priman los frames de confrontación (conflicto), de responsabilidad y de moralidad, mientras que los frames menos frecuentes son los de peligro y de diálogo (ver la figura 4).

Si contrastamos estos resultados con las elecciones municipales del 2014, encontramos que los frames de confrontación, de responsabilidad y de moralidad se mantienen en los primeros tres puestos. Vemos entonces que la tendencia es consistente entre las dos elecciones, a pesar de las diferencias de las muestras. Esto expresa, además, que existen ciertos patrones y reglas de producción periodística para cubrir procesos electorales, que se mantienen a pesar de tratarse de elecciones sustantivamente distintas.

Figura 3. Frame de nivel según primera y segunda vuelta electoral

\begin{tabular}{|l|c|c|c|c|c|c|}
\hline & \multicolumn{2}{|c|}{ Primera vuelta } & \multicolumn{2}{c|}{ Segunda vuelta } & \multicolumn{2}{c|}{ Total } \\
\hline & Frec. & $\%$ & Frec. & $\%$ & Frec & $\%$ \\
\hline Frames episódicos & 1330 & 43.6 & 828 & 40.7 & 2158 & 42.4 \\
\hline Frames de asuntos & 1539 & 50.5 & 1126 & 55.3 & 2665 & 52.4 \\
\hline Frame de políticas & 181 & 5.9 & 82 & 4.0 & 263 & 5.2 \\
\hline & 3050 & 100.0 & 2036 & 100.0 & 5086 & 100.0 \\
\hline
\end{tabular}

Significación = 0.00 (Chi cuadrado de Pearson).

Figura 4. Frames genéricos según proceso electoral

\begin{tabular}{|l|l|l|l|l|}
\hline & \multicolumn{2}{|c|}{ Presidenciales 2016 } & \multicolumn{2}{c|}{ Municipal 2014 } \\
\hline & Frec. & $\%$ & Frec. & $\%$ \\
\hline Frame de confrontación & 1854 & 36.3 & 69 & 33.0 \\
\hline Frame de responsabilidad & 1027 & 20.1 & 20 & 9.6 \\
\hline Frame de moralidad & 818 & 16.0 & 39 & 18.7 \\
\hline Frame de interés humano & 772 & 15.1 & 7 & 3.4 \\
\hline Frame de consecuencias económicas & 528 & 10.3 & 11 & 5.6 \\
\hline Frame de peligro & 407 & 8.0 & 11 & 5.3 \\
\hline Frame de diálogo & 432 & 8.5 & 22 & 10.5 \\
\hline & $\mathrm{n}=5112$ & & $\mathrm{n}=209$ & \\
\hline
\end{tabular}


La alta cifra de frames de conflictos nos muestra que las elecciones son enmarcadas como confrontaciones, exacerbando las diferencias y oposición entre los candidatos, lo que abona en la idea de que la cobertura tiende a centrarse en lo estratégico, viendo quién gana y quién pierde. Así mismo, es interesante la presencia sustantiva del frame de moralidad, es decir, que los medios juzgan y adjetivan los discursos o las acciones de los actores políticos en las elecciones, lo que podría estar abonando en el distanciamiento político de los electores respecto a los candidatos.

Comparando el uso de los frames genéricos en primera y segunda vuelta hemos observando diferencias interesantes. En primer lugar, no hay diferencias significativas en el uso del frame de confrontación (conflicto) y de moralidad entre ambos periodos. De manera que la disputa y el juicio son elementos discursivos sustantivos en la forma de cubrir los procesos electorales, no viéndose afectados por los procesos que supone el diseño electoral de primera o segunda vuelta (ver la figura 5).

Por el contrario, el frame de interés humano tiene una mayor presencia en la segunda vuelta electoral, posiblemente para vincular las demandas de la población con las propuestas de los candidatos. En cambio, el frame de consecuencias econ'omicas se duplica de primera a segunda vuelta, creemos porque en esta última hay una concentración en los problemas que se generarían de ganar uno $\mathrm{u}$ otro candidato.

Así mismo, el frame de consecuencias econ'omicas tiene un significativo incremento de primera a segunda vuelta probablemente debido al esfuerzo por deslegitimar una candidatura por sobre otra. Hay que considerar que la segunda vuelta se realiza entre dos candidatos, lo que naturalmente implica un proceso de polarización donde los discursos buscan

Figura 5. Frames genéricos según primera y segunda vuelta electoral

\begin{tabular}{|l|c|c|c|c|c|c|}
\hline & \multicolumn{2}{|c|}{ Primera vuelta } & Segunda vuelta & \multicolumn{4}{|c|}{ Total } \\
\hline & Frec. & $\%$ & Frec. & $\%$ & Frec. & $\%$ \\
\hline Frame de confrontación & 1126 & 36.8 & 728 & 35.5 & 1854 & 36.3 \\
\hline Frame de responsabilidad** $^{*}$ & 661 & 21.6 & 366 & 17.9 & 1027 & 20.1 \\
\hline Frame de moralidad & 484 & 15.8 & 334 & 16.3 & 818 & 16.0 \\
\hline Frame de interés humano** $^{*}$ & 419 & 13.7 & 353 & 17.2 & 772 & 15.1 \\
\hline Frame de consecuencias económicas** $^{*}$ & 212 & 6.9 & 316 & 15.4 & 528 & 10.3 \\
\hline Frame de peligro & 233 & 7.6 & 174 & 8.5 & 407 & 8.0 \\
\hline Frame de dialogo* & 239 & 7.8 & 193 & 9.4 & 432 & 8.5 \\
\hline
\end{tabular}

** Significativo al 0.01. *Significativo al 0.05 (en base a la prueba exacta de Fisher). 
favorecer a uno de ellos y desfavorecer al otro. Proceso en el cual los medios se ven envueltos.

En la figura 6 observamos que seis de los siete frames genéricos presentan diferencias significativas según el tipo de medio. Los frames de confrontación y de responsabilidad son más usados, proporcionalmente, en prensa que en el resto de medios. En cambio, los frames de moralidad y de consecuencias económicas son los más empleados en la radio. La televisión, por su parte, emplea una variedad de frames sin diferenciarse respecto al resto de tipos de medios (ver la figura 6).

Los resultados presentados van en contra del sentido común que suele asociar a la televisión con la confrontación, siendo, en cambio, la prensa que, de todos los frames que emplea, es el de confrontación el que está más presente en sus noticias. Así mismo, se esperaría que la prensa fuera el medio que más uso hace del frame de consecuencias económicas, toda vez que suele tener periodistas especializados y secciones dedicadas el tema económico. Sin embargo, es la radio la que usa con más frecuencia este frame. La radio también es el medio que más uso hace del frame de diálogo, imaginamos debido a su rol de articulador su mayor interacción con público, invitados y expertos que transitan por el medio.

\section{Conclusiones}

5.1 Este estudio nos muestra que existen algunas tendencias en la producción periodística de los frames que se mantienen de una elección a otra, especialmente en los frames genéricos. Esta continuidad se basa especialmente en el uso de la confrontación, el juicio moral y la atribución de responsabilidad.

El caso peruano es coherente con otros estudios donde predominan el uso de

Figura 6. Frames genéricos según tipo de medio

\begin{tabular}{|l|l|l|l|l|l|l|}
\hline & \multicolumn{2}{|c|}{ Radio } & \multicolumn{2}{|c|}{ Televisión } & \multicolumn{2}{c|}{ Prensa } \\
\hline & Frec. & $\%$ & Frec. & $\%$ & Frec. & $\%$ \\
\hline Frame de confrontación $^{* *}$ & 219 & 28.9 & 1101 & 35.5 & 534 & 42.7 \\
\hline Frame de responsabilidad** & 161 & 21.2 & 472 & 15.2 & 394 & 31.5 \\
\hline Frame de moralidad** $^{*}$ & 149 & 19.7 & 444 & 14.3 & 225 & 18.0 \\
\hline Frame de interés humano & 129 & 17.0 & 460 & 14.8 & 183 & 14.6 \\
\hline Frame de consecuencias económicas** & 150 & 19.8 & 178 & 5.7 & 200 & 16.0 \\
\hline Frame de peligro** & 35 & 4.6 & 276 & 8.9 & 96 & 7.7 \\
\hline Frame de dialogo** & 99 & 13.1 & 259 & 8.3 & 74 & 5.9 \\
\hline
\end{tabular}

** Significativo al 0.01. * Significativo al 0.05 (Chi cuadrado de Pearson) 
frames de conflicto o confrontación. Esto ha sido observado en elecciones de otros países como Estados Unidos y Suecia (Dimitrova y Strömbäck, 2011), o en México (Muñiz et al., 2018). Sin embargo, en otros países como Holanda, Semetko y Valkenburg (2000) encuentran que predomina el frame de atribución de responsabilidad por encima del de conflicto, seguidos del frame económico. Esto indica que la configuración de la cobertura -respecto a los frames- puede depender de las culturas periodísticas nacionales.

La particularidad de la cobertura peruana de las elecciones radica en la predominancia del frame de moralidad, pues este, según los estudios internacionales revisados, es un frame que se usa muy poco para procesos electorales. La incorporación del frame de moralidad en la producción periodística de una elección a otra puede deberse al rol regulador que asume el periodismo ante la debilidad de los partidos políticos. Sin embargo, hay que considerar que la cultura de producción periodística puede ser influenciada por una cualidad cultural de la sociedad peruana, basada en prácticas religiosas sancionadoras y punitivas.

5.2 Otro aspecto que resalta de este estudio es que el uso de los frames responde a las coyunturas electorales, en particular a como están organizadas nuestras elecciones. La configuración de dos vueltas electorales con muchas candidaturas al inicio modo particular del uso de los frames para dar cuenta de condiciones diferentes.

La literatura internacional muestra que el uso de frames variaba sustantivamente ante controversias diferentes, como por ejemplo en la incorporación del euro y en la discusión de políticas económicas o de privatización (de Vreese et al., 2001; Semetko y Valkenburg, 2000; Aruguete, 2010). Pero en este estudio hay frames como los de conflicto y los de moralidad que se mantienen predominantes en las dos etapas, posiblemente debido a la tendencia a cubrir las elecciones como "carreras de caballos" y al rol de gatekeeper que los medios asumen en resguardo del modelo económico liberal. Otros frames como los de interés humano y los de responsabilidad cambian según las situaciones que configuran los periodos electorales.

En el caso peruano encontramos que el frame de conflicto está presente en ambas etapas. Más aún, Muñiz et al. (2018) encuentran que conforme se acerca la fase final de la campaña electoral hay un uso más intensivo de este tipo de frame. Es probable que la diferencia con otras coberturas radique en que en el caso mexicano, por ejemplo, se trata de una campaña más corta (de solo cuatro semanas frente a las ocho del caso peruano), por tanto, la adecuación de los medios al proceso electoral debe ser diferente. En todo caso, los resultados encontrados respecto a otros frames y el estudio de Muñiz et al. (2018) coinciden en que en los análisis de 
los frames en periodos electorales es primordial la realización de longitudinales.

5.3 En el caso peruano, el tipo de medio no marca la diferencia en la producción de frames de conflicto. En este estudio hallamos usos de frames que no siguen la discusión que asocia la televisión al conflicto. En un estudio en 27 países de la Unión Europea, Schuck et al. (2013) encuentra que la diferencia entre prensa y televisión tampoco influye en la predominancia del frame de conflictos. Una conclusión opuesta llega el estudio de Muñiz et al. (2018) que encuentra que hay una diferencia sustantiva en el uso de frames entre la televisión y la prensa, pues en la primera hay una mayor presencia del frame de conflicto.

Los otros estudios revisados no comparan los otros frames genéricos en elecciones, por lo que es difícil concluir si es un patrón el uso en procesos electorales de los frames de conflicto o es extensivo a los otros frames. El estudio de Semetko y Vankelburke (2000), por su parte, si bien sí compara cinco tipos de frames genéricos y encuentra diferencias entre prensa y televisión en los frames de interés humano y en los de consecuencias económicas, el estudio se realizó en un periodo no electoral.

El presente estudio encuentra que los medios peruanos hacen uso de los otros frames de manera diferencial, rompiendo con la generalidad de que la cobertura de las elecciones se da por igual en todos los medios.

5.4 En cuanto a la pregunta sobre si la cobertura peruana de las elecciones sigue un patrón similar al estilo "americano", encontramos que al menos en la fase de segunda vuelta los medios de comunicación no se guían por la "carrera de caballos", probablemente debido al efecto que los debates presidenciales tienen en relevar lo temático en la última semana de elecciones.

Más bien coincidimos con Esser y Strömbäck (2012) en que la cobertura de medios en algunos países, como el Perú, se trata de modelos híbridos, donde se usa predominantemente el frame de conflicto al mismo tiempo que se incorpora el frame de moralidad (que la literatura no observa como de uso predominante en procesos electorales), y que estos, sumados al empleo del frame de responsabilidad, conforman una cobertura con mucha adjetivación.

Esta particularidad puede deberse el rol de gatekeeper que en el Perú desarrollan los medios de comunicación en la medida en que la representación política y el sistema de partidos se encuentran colapsados, erigiéndose estos como los jueces de las candidaturas que consideran lesivas al orden imperante.

Al mismo tiempo observamos que se mantienen los patrones internacionales 
de cobertura de elecciones, focalizándose en el conflicto, poniendo poca atención a los frames de políticas, lo que lleva a desatender la necesidad pública de deliberación de las directrices de la gobernanza futura que toda elección debe abordar.

\section{REFERENCIAS}

Aruguete, N. (2010). Los encuadres noticiosos en los medios argentinos. Un análisis de la privatización de Entel. América Latina Hoy, 54, 113-137.

Bartholome, G.; Lecheler, S. y de Vreese, C. (2017). Towards a Typology of Conflict Frames. Substantiveness and interventionism in political conflict news. Journalism Studies, 19 (12), 1689-1711. https://doi.org/10.1080/14 61670X.2017.1299033

Busby, E.; Flynn, D. y Druckman, J. (2018). Studying Framing Effects on Political Preferences: Existing Research and Lingering Questions. En P. D’Angelo (Ed), Doing framing analysis II. Empirical and theoretical perspectives (pp. 67-90). New York: Routlegde.

Blumler, J. y Coleman, S. (2010). Political Communication in freefall: The British case and Others? International Journal of Press/Politics, 15 (2), 139-154. https://doi. org/10.1177/1940161210362263

Capella, J. y Jamieson, K. (1997). Spiral of cynicism. The press and the public good. Oxford: Oxford University Press

Chong, D. y Druckman, J. (2007). Framing Theory. Annual Review of Political Science, 10 (1), 103-126. https:// doi.org/10.1146/annurev.polis-
D’Angelo, P. (2018). A Typology of Frames in News Framing Analysis. En P. D’Angelo (Ed), Doing framing analysis II. Empirical and theoretical perspectives (pp. 23-40). New York: Routledge. https://doi. org/10.4324/9781315642239

De Vreese, C. (2005). News framing: Theory and typology. Information Design Journal, 13 (1), 51-62. https://doi. org/10.1075/idjdd.13.1.o6vre

De Vreese, C.; Peter, J. y Semetko, H. A. (2001). Framing politics at the launch of the euro: A cross-national comparative study of frames in the news. Political Communication, 18 (2), 107-122. https://doi. org/10.1080/105846001750322934

Dimitrova, D. y Kostadinova, P. (2014). Identifying Antecedents of the Strategic Game Frame: A longitudinal analysis. Journalism \& Mass Communication Quarterly, 9o (1), 75-88. https:// doi.org/10.1177/1077699012468739

Dimitrova, D. y Strömbäck, J. (2011). Election news in Sweden and the United States: A comparative study of sources and media frames. Journalism, 13 (5), 604-619. https://doi. org/10.1177/1464884911431546

Esser, F. y Strömbäck, J. (2012). Comparing News on National Elections. En F. Esser y T. Hanitzsch (Eds.), Handbook of Comparative Communication Research (pp. 308-326). Londres: Routledge.

Entman, R. (1993). Framing: Toward clarification of a fractured paradigm. Journal of Communication, 43 (4), 51-58. https://doi. org/10.1111/j.1460-2466.1993. tbo1304.X

Entman, Robert. (1991). Framing U.S. Coverage of International News: Contrast in narratives of the Kal and 
Iran Incidents. Journal of Communication, 41 (4), 6-26. https:// doi.org/10.1111/j.1460-2466.1991. tbo2328.x

Gamson, W. y Modigliani, A. (1989). Media discourse and public opinion on nuclear power: A constructionist approach. American Journal of Sociology, 95 (1), 1-37. https://doi. $\operatorname{org} / 10.1086 / 229213$

Gamson, W.; Ccroteau, D.; Honey, W. y Sansom, T. (1992). Media images and the social construction of reality. Annual Review of Sociology, 18, 373-393. https://doi.org/10.1146/annurev. so.18.080192.002105

Gitlin, T. (1980). The Whole World is Watching: Mass Media in the Making \& Unmaking of the New Left. Berkeley: University of California Press.

Goffman, E. (1986). Frame Analysis. An Essay on the Organization of Experience. Boston: Northeastern University Press.

Hakansson, N. (2000). Framing the Tunnel. Local news media and the Hallandsas Toxic Leak 1997. Gotemburgo: CEFOS. Göteborgs Universitet.

Iyengar, S. (1994). Is anyone responsible? How television frames political issues. Chicago: University of Chicago Press.

Kiousis, S. (2005). Compelling Arguments and Attitude Strength. Exploring the Impact of Second-Level Agenda Setting on Public Opinion of Presidential Candidate Images. Harvard International Journal of Press/ Politics, 10 (2), 3-27. https://doi. org/10.1177/1081180X05276095

Kozman, C. (2016). Measuring Issue-Specific and Generic Frames in the Media's Coverage of the Steroids Issue in Baseball, Journalism Practice, 11, 777-797 https://doi.org/10.1080/17512 786.2016.119066o
Macassi, S. y Graham, L. (2003). Termómetro Mediático $n^{\circ} 4$. Lima: Centro de Investigación A.C.S. Calandria. Recuperado de https://bit.ly/2KleLwR

Manin, B. (2006). Los principios del gobierno representativo. Madrid: Alianza ed.

Matther, J. (2012). Framing Politics: An Integrative Approach. American Behavioral Scientist, 56 (3), 300-317. https://doi. org/10.1177/0002764211426324

Muñiz, C. (2015). La política como debate temático o estratégico. Framing de la campaña electoral mexicana de 2012 en la prensa digital. Comunicación y Sociedad, (23), 67-94. https://doi. org/10.32870/cys.voi23.64

Muñiz, C.; Saldierna, A. R. y Marañón, F. J. (2018). Framing of electoral processes: The stages of the campaign as a moderator of the presence of political frames in the news, Palabra Clave, 21 (3), 740-771. https://doi.org/10.5294/ pacla.2018.21.3.5

Neuman, R.; Just, M. y Crigler, A. (1992). Common knowledge. Chicago: University of Chicago Press. https://doi.org/10.7208/chicago/9780226161174.001.0001

Nicolás, M. T. (2011). Framing the news: from political conflict to peace. How the 'framing theory' and the 'political context model' can enhance the peace journalism model. Journal of Latin American Communication Research, 1 (2), 48-61.

Patterson, T. (1994). Out of Order: An incisive and boldly original critique of the news media's domination of America's political process. New York: Vintage book edition.

Programa de las Naciones Unidas para el Desarrollo (PNUD). (2004). La democracia en América Latina. Hacia una democracia de ciudadanas y ciudadanos. Buenos Aires: Taurus. 
Schmuck, D.; Heiss, R.; Matthes, J.; Engesser, S. y Esser, F. (2016). Antecedents of strategic game framing in political news coverage. Journalism, 18 (8), 937-955. https://doi. org/10.1177/1464884916648098

Semetko, H. y Valkenburg, P. (200o). Framing European politics: a content analysis of press and television news. Journal of communication, 40 (2), 93-109. https://doi. org/10.1111/j.1460-2466.2000. tbo2843.x

Scheufele, D. A. (1999). Framing as a theory of media effects. Journal of Communication, 49 (4), 103-122. https:// doi.org/10.1111/j.1460-2466.1999. tbo2784.X

Scheufele, D. e Iyengar, S. (2017). The state of framing research: A call for new directions. En K. Kenski y K. H. Jamieson (Eds.), The Oxford Handbook of Political Communication. Oxford: Oxford University Press.

Strömbäck, J. (2008). Four phases of mediatization: An analysis of the mediatization of politics. The International Journal of Press/Politics, 13 (3), 228-246. https://doi. org/10.1177/1940161208319097

Schuck, A. et al. (2013). Explaining Campaign News Coverage: How Medium, Time, and Context Explain Variation in the Media Framing of the 2009 European Parliamentary Elections. Journal of Political Marketing, 12 (1), 8-28. https://doi.org/10.1080/15377857.201 3.752192

Tuchman, G. (1973). Making News by Doing Work: Routinizing the Unexpected. American Journal of Sociology, 79 (1), 110-131. https://doi. org/10.1086/225510 